\title{
Air Leak Syndrome in Two Very Preterm Infants Born to Mothers with Coronavirus Disease 2019: An Association or a Coincidence?
}

\author{
Ajay Reddy, MD ${ }^{1}$ Krystin Engelhardt, DO $^{1}$ Deepak Jain, MD ${ }^{1}$ (1) \\ ${ }^{1}$ Division of Neonatology, Department of Pediatrics, Rutgers \\ University, Robert Wood Johnson Medical School, New Brunswick, \\ New Jersey \\ Address for correspondence Deepak Jain, MD, Department of \\ Pediatrics, Rutgers, The State University of New Jersey, MEB - room \\ no: 396, 1, Robert Wood Johnson Palace, New Brunswick, NJ 08901 \\ (e-mail: dj392@rwjms.rutgers.edu).
}

Am J Perinatol Rep 2020;10:e266-e269.

\begin{abstract}
The novel coronavirus disease 2019 (COVID-19) caused by the severe acute respiratory syndrome coronavirus 2 (SARS-CoV-2) mostly affects adults with limited information on possible vertical transmission from pregnant mothers. We present here two very preterm infants born to mothers with COVID-19, whose respiratory course was significant for initial mild respiratory distress syndrome who developed acute onset severe air leak syndrome at approximately 24 to 36 hours of age requiring thoracentesis. Their respiratory status improved gradually with resolution of air leak and respiratory failure by 2 weeks of age. Both infants tested negative for SARS-CoV-2 by reverse transcriptase-polymerase chain reaction of multiple respiratory specimens collected beyond 24 hours after birth. As the incidence of severe air leak syndrome is relatively low in preterm infants without risk factors, this

Keywords

- COVID-19

- coronavirus

- SARS-CoV-2

- pneumothorax

- preterm presentation in two very preterm infants born to mothers with COVID-19 is intriguing and needs to be further evaluated in larger cohorts. If confirmed, this data could potentially be the first step toward generating hypotheses for mechanisms of lung injury, intrauterine transmission, or how to detect COVID-19 in preterm infants. In addition, these data will be critical for developing evidence-based guidelines for perinatal management of these infants as we continue to battle against the COVID-19 pandemic for the foreseeable future.
\end{abstract}

The novel coronavirus disease 2019 (COVID-19) is a potentially life-threatening condition caused by the severe acute respiratory syndrome coronavirus 2 (SARS-CoV-2) that has rapidly spread across the globe and presented itself as a significant public health problem. There is limited data on whether the clinical course of COVID-19 in pregnant women is different from nonpregnant adults, with some evidence that it may not be associated with poor maternal or perinatal outcomes. The majority of the women in these studies presented in third trimester with delivery at late preterm or term, and neonates had unremarkable hospital course with no clear evidence of vertical transmission. ${ }^{1-3}$

There is very limited data on the clinical course of preterm infants of less than 32 weeks of gestation (very preterm) born

received

May 31, 2020

accepted

June 23,2020
DOI https://doi.org/

$10.1055 / \mathrm{s}-0040-1715180$. ISSN 2157-6998. to mothers with SARS-CoV-2 infection, thereby presenting a significant challenge to the management of these infants. ${ }^{4,5}$ We report here two very preterm infants born to mothers with COVID-19 with an atypical respiratory course complicated by air leak syndrome.

\section{Case 1}

Patient was a female infant born at $29^{3 / 7}$ weeks of gestation with a birthweight of $1,490 \mathrm{~g}$. She was born to a 37 -year-old gravida 6 , para 4 mother with history of gestational diabetes on metformin and Class II obesity.

Maternal history was significant for cough, sore throat, body ache, chills, frontal headache, and abdominal pain 10 days prior

Copyright @ 2020 by Thieme Medical Publishers, Inc., 333 Seventh Avenue, New York, NY 10001, USA. Tel: +1(212) 760-0888.
License terms

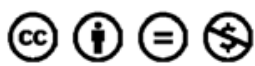


to delivery, and she tested positive for SARS-CoV-2 on reverse transcriptase-polymerase chain reaction (RT-PCR) by nasopharyngeal (NP) swab. Her other prenatal laboratories were unremarkable. She was observed for 1 day and was discharged home. She presented 2 days later in the emergency department (ED) with increasing complaints of headache, body aches, and contractions, and was admitted for observation for 24 hours. During this period, her examination remained unchanged, with intact membranes, stable respiratory status, and a reactive nonstress test. She was discharged home with follow-up in high-risk clinic. She presented 2 days later in preterm labor and noted to be fully dilated with crowning of head on exam. She went on to have precipitous delivery of a female infant. Mother did not receive antenatal steroids.

The neonatal resuscitation team followed airborne isolation precautions, and cord was clamped immediately after birth due to maternal COVID-19 status. Patient was vigorous at birth and required continuous positive airway pressure (CPAP) of $5 \mathrm{~cm}$ of $\mathrm{H}_{2} \mathrm{O}$ and $\mathrm{FiO}_{2}$ of 0.3 . Apgar's scores were 8 and 8 at 1 and 5 minutes, respectively. She was admitted to the neonatal intensive care unit (NICU) in a negative pressure isolation room with airborne precautions and started on CPAP of $6 \mathrm{~cm}$ of $\mathrm{H}_{2} \mathrm{O}$ and $\mathrm{FiO}_{2}$ of 0.3 . Initial chest radiograph (CXR) was suggestive of respiratory distress syndrome (RDS) ( - Fig. 1A), and her initial capillary blood gas showed $\mathrm{pH}$ of 7.3, $\mathrm{PCO}_{2} 51, \mathrm{PO}_{2} 44$, and base excess 1.1. Infant was started on caffeine prophylaxis for apnea of prematurity, and empiric ampicillin and gentamicin due to preterm labor. She continued to be on CPAP of $6 \mathrm{~cm}$ of $\mathrm{H}_{2} \mathrm{O}$ and $\mathrm{FiO}_{2}$ of 0.25 to 0.3 until approximately 20 hours of age when she had acute respiratory decompensation with recurrent episodes of apnea, bradycardia, and desaturations requiring increase in oxygen supplementation. She was intubated and given surfactant with some improvement in oxygen requirement. However, the CXR performed for tube position showed left-sided tension pneumothorax (-Fig. 1B) which required needle thoracentesis followed by chest tube placement. Over the next few days, the infant developed diffuse pulmonary interstitial emphysema, subcutaneous emphysema, and a right-sided pneumothorax requiring right chest tube placement (-Fig. 1C). Her respiratory status gradually improved, and she was extubated on day 5 to CPAP, and the chest tubes were removed by day 10 . Follow-up CXRs denoted complete resolution of air leaks. The infant was weaned to nasal cannula on day 11 and weaned to room air on day 17.

RT-PCR performed on NP swab at 24 hours and repeat NP swab and tracheal aspirate at 60 hours of age were negative for SARS-CoV-2. A respiratory viral panel was negative. Infant received antibiotics for 10 days for presumed pneumonia. Her clinical course was remarkable for development of a left grade 4 and right grade 2 intraventricular hemorrhage.

After delivery, mother developed COVID-19 pneumonia and required nasal cannula oxygen. Hydroxychloroquine was added to the medication regimen on postpartum day 3. Her respiratory status improved, and mother was discharged on postpartum day 5 .

\section{Case 2}

Patient was a male infant born at $29^{3 / 7}$ weeks of gestation with a birthweight of $1,220 \mathrm{~g}$. Infant was born to a 28 -year-old gravida 4, para 3 mother with antenatal history significant for chronic hypertension, chronic kidney disease, and obesity.

The mother presented to the ED 12 days prior to delivery with fever, cough, sore throat, and shortness of breath. She tested positive for SARS-CoV-2 on RT-PCR of NP swab, and was admitted for monitoring due to COVID-19 superimposed on worsening preeclampsia. Her other prenatal laboratories were unremarkable. During her hospital course, she received labetalol, magnesium, and a course of betamethasone 11 days prior to delivery. Due to worsening preeclampsia and COVID-19 pneumonia, caesarian section was performed at 29 weeks $^{3 / 7}$ of gestation. At the time of delivery, she was on high-flow nasal cannula oxygen and prophylactic anticoagulation with heparin due to the risk for thrombosis in pregnancy and COVID-19.

Upon delivery, the neonatal team followed airborne precautions during resuscitation, and cord was clamped immediately after delivery. The infant was vigorous at birth and did not require any respiratory support with Apgar's scores of 9 and 9 at 1 and 5 minutes, respectively. The infant was transferred to the NICU in room air and admitted to a negative pressure isolation room due to maternal COVID-19. Within few hours of birth, infant was noted to have increased work of breathing and was placed on nasal CPAP of $6 \mathrm{~cm}$ of $\mathrm{H}_{2} \mathrm{O}$ with and $\mathrm{FiO}_{2}$ of 0.25

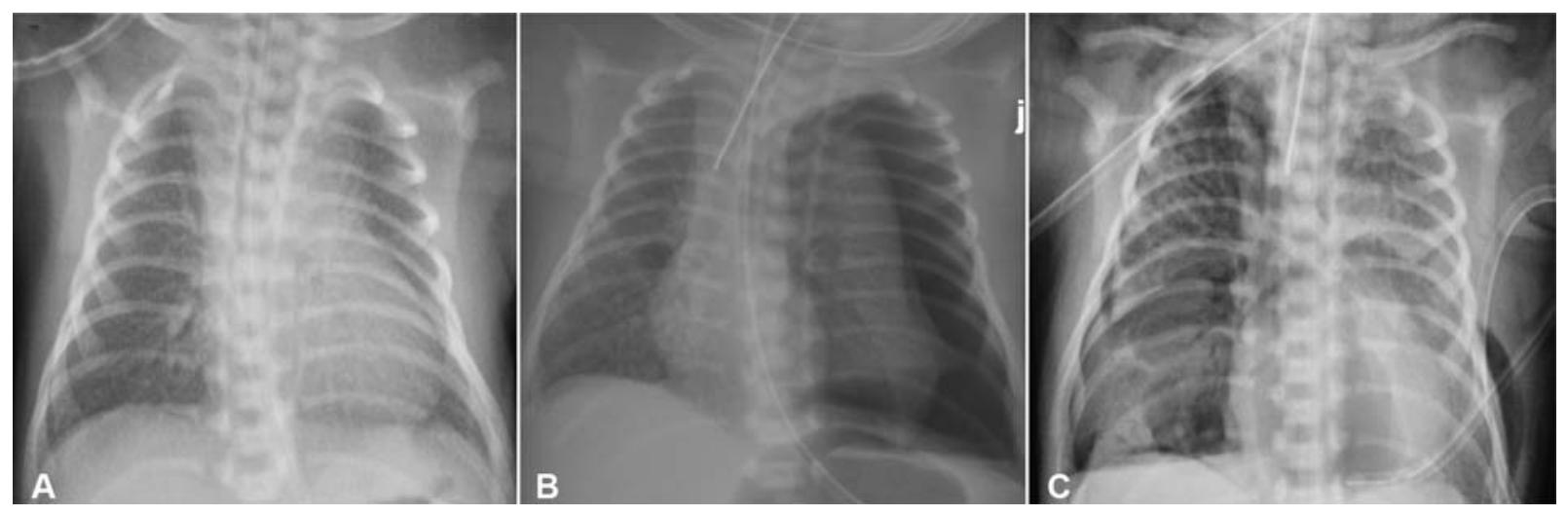

Fig. 1 Series of chest radiographs from the first case with initial image showing diffuse ground glass pattern (A), a large left pneumothorax on second image (B), and the third image showing right pneumothorax, pulmonary interstitial emphysema, and subcutaneous emphysema (C). 

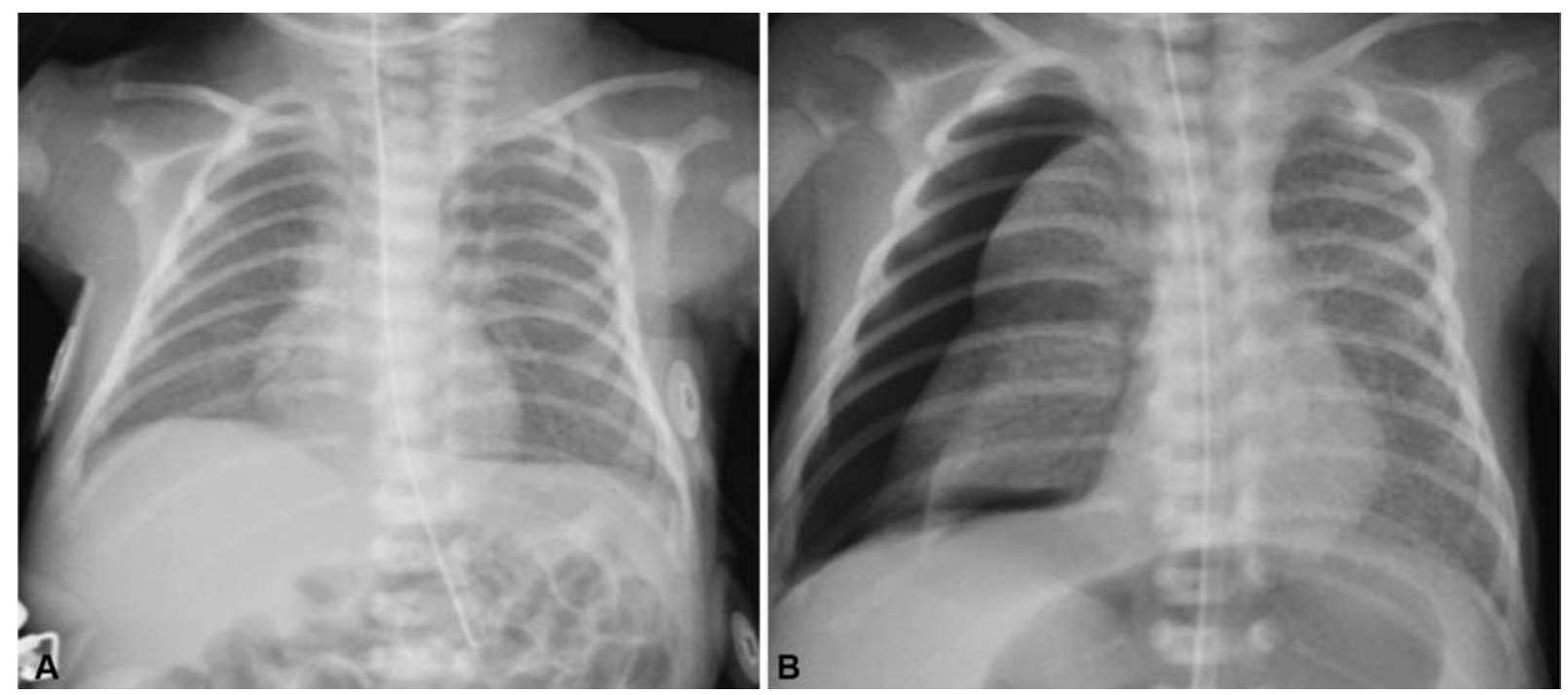

Fig. 2 Series of chest radiographs from the second case with initial image showing diffuse ground glass pattern (A), and a large right pneumothorax on the second image (B).

to 0.3. CXR at this time was suggestive of RDS ( - Fig. 2A), and a capillary blood gas showed $\mathrm{pH}$ of $7.38, \mathrm{PCO}_{2} 43, \mathrm{PO}_{2} 44$, and a base excess of 0.5 . At approximately 36 hours of age, infant had sudden deterioration with increased work of breathing and oxygen requirement, and CXR showed a right sided tension pneumothorax ( $\mathbf{- F i g}$. 2B). Needle thoracentesis followed by right-sided chest tube placement was performed immediately with improvement in pneumothorax. The infant had to be intubated 16 hours after chest tube placement for recurrent apnea episodes and increase in oxygen requirement. Over next few days, ventilator settings were gradually weaned, and he was extubated to CPAP on day 6. Chest tube was removed on day 9 and he was weaned off respiratory support by day 14 .

Repeated testing for SARS-CoV-2 on RT-PCR by NP swab at 36 and 72 hours and tracheal aspirate at day 6 were negative. The infant reached full enteral feeds by day 9 . Serial head ultrasounds obtained on day 3 and 7 have been unremarkable.

\section{Discussion}

We report the perinatal and early neonatal course of two very preterm infants born to mothers with COVID-19. Both of these infants followed similar respiratory courses with initial mild RDS complicated by development of severe air leak syndrome around 24 to 36 hours after birth requiring chest tube placement. Both infants tested negative for SARS-CoV-2 by RT-PCR of multiple respiratory specimens collected beyond 24 hours after birth.

Although pneumothorax is not a rare diagnosis in the $\mathrm{NICU}$, its incidence is relatively low in very preterm infants without any risk factors. ${ }^{6}$ Some of the factors associated with the development of pneumothorax in premature infants include pulmonary hypoplasia, severe RDS, or use of high level of CPAP during resuscitation at birth. ${ }^{7}$ Both infants in the current case report had mild RDS on initial CXR, low oxygen requirement, and adequate ventilation on CPAP of 5 to $6 \mathrm{~cm}$ of $\mathrm{H}_{2} \mathrm{O}$ before acute deterioration. The respiratory status of both cases improved rapidly after chest tube placement, and the infants were weaned from respiratory support within 2 weeks. Whether this atypical respiratory course in the setting of maternal COVID-19 reflects an insult to the developing lung needs to be further explored in larger studies.

The inconsistency in the use of ANS between the two cases in the current case series reflects the lack of evidence and ongoing debate on their use in mothers with SARS-CoV-2 infection. It is plausible that the lack of exposure to ANS in the first case contributed to the air leak syndrome; however, it is important to note that though ANS have been shown to reduce the risk of RDS, the studies have failed to show any reduction in the incidence of air leak syndrome. ${ }^{8}$ While the Centers for Disease Control and Prevention recommends against the use of corticosteroids in general population with COVID-19 due to possible risk of prolonged viral replication, it has not provided any guidelines specific for ANS. On the other hand, American College of Obstetricians and Gynecologists have continued to recommend ANS for mother with SARS-CoV-2 infection at less than 34 weeks of gestation while recommending against their use beyond 34 weeks of gestation. ${ }^{9}$ These inconsistencies reflect an urgent need for studies to evaluate the effects of ANS not only on the maternal SARS-CoV-2 infection, but also on the fetal organ development to develop guidelines with a stronger evidence base. ${ }^{10}$

There is very limited information on the clinical presentation of SARS-CoV-2 in the neonatal period for multiple reasons. These include the low incidence of positive NP RT-PCR and the overlap between common clinical manifestations of COVID-19 in adults and respiratory conditions affecting neonates at birth, such as transient tachypnea of newborn or RDS. ${ }^{3,11}$ of note, in one of the few described cases of neonates with positive SARS-CoV-2, Coronado et al described a 3-week-old infant with late-onset sepsis complicated by the development of pneumothorax. ${ }^{12}$ 
None of the infants in this case report were positive for SARS-CoV-2 by RT-PCR of respiratory specimen. One of the shortcomings of the current case series is that we did not evaluate respiratory specimen for any bacteria. In addition, we could not evaluate any other specimen such as amniotic fluid, placenta, stool, or cord blood for presence of virus or serum antibody testing, thereby limiting the ability to comment on possibility of vertical transmission. The sensitivity and specificity of respiratory specimen to detect transplacental transmission has not been fully evaluated. In one recent case series, infants born to mothers with COVID-19 pneumonia had positive serum IgG and IgM with negative RT-PCR tests. ${ }^{13}$ In another study of adults evaluating SARS-CoV-2 in different clinical specimens, broncheoalveolar lavage fluid had the highest positive rate; in addition to virus being positive in sputum, nasal swab, stool, blood, and urine. ${ }^{14}$ These reports do raise the question as to whether a negative RT-PCR from a respiratory specimen in at-risk infants should be considered as an absence of infection, and whether there is a need for more comprehensive testing in these infants. ${ }^{15,16}$

The evidence for vertical transmission of SARS-CoV-2 has been sparse, with most of the case series reporting no neonates with positive RT-PCR in the respiratory specimen. Most of this data are from late-term or full-term neonates born to asymptomatic mothers or those with mild symptoms. $^{2,17}$ There is increasing evidence that this may not hold true in neonates born at earlier gestations or to mothers with severe COVID-19, with recent case reports suggesting possible vertical transmission in infants born to mothers with severe COVID-19 illness. $^{18,19}$

As evidence accumulates for the effect of maternal COVID19 in preterm infants, majority of the focus has been on transmissibility of the virus from mother to the neonate around the time of birth. Some of the questions remaining center on the transplacental transfer of virus during early part of gestation and the potential effect of maternal COVID19 on the development of the fetal lungs and other organ systems. The observational data from case reports like the one presented here, if replicated in larger cohorts, could potentially be the first step toward generating hypotheses to be tested in the preclinical or clinical models. The resultant data will be key to develop evidence-based perinatal care strategies like use of ANS, delayed cord clamping, or choice of respiratory support after birth. ${ }^{20,21}$

\section{Conclusion}

We describe two very preterm infants born to mothers with COVID-19 with early respiratory course complicated by severe air leak syndrome. These observations need to be further investigated in larger cohorts and if confirmed may have significant implications on perinatal management of these infants as we continue to battle against the COVID-19 pandemic for the foreseeable future.

Conflict of Interest

None declared.

\section{References}

1 Breslin N, et al. COVID-19 infection among asymptomatic and symptomatic pregnant women: two weeks of confirmed presentations to an affiliated pair of New York City hospitals. Am J Obstet Gynecol MFM 2020:100118

2 Elshafeey F, Magdi R, Hindi N, et al. A systematic scoping review of COVID-19 during pregnancy and childbirth. Int J Gynaecol Obstet 2020;150(01):47-52

3 Zeng L, et al. Neonatal Early-Onset Infection With SARS-CoV-2 in 33 Neonates Born to Mothers With COVID-19 in Wuhan, China. JAMA Pediatr 2020;174(07):722-725

4 Juan J, Gil MM, Rong Z, Zhang Y, Yang H, Poon LC. Effect of coronavirus disease 2019 (COVID-19) on maternal, perinatal and neonatal outcome: systematic review. Ultrasound Obstet Gynecol 2020;56(01):15-27

5 Piersigilli F, Carkeek K, Hocq C, et al. COVID-19 in a 26-week preterm neonate. Lancet Child Adolesc Health 2020;4(06):476-478

6 Vibede L, Vibede E, Bendtsen M, Pedersen L, Ebbesen F. Neonatal pneumothorax: a descriptive regional danish study. Neonatology 2017;111(04):303-308

7 Morley CJ, Davis PG, Doyle LW, Brion LP, Hascoet JM, Carlin JB; COIN Trial Investigators. Nasal CPAP or intubation at birth for very preterm infants. N Engl J Med 2008;358(07):700-708

8 Roberts D, Brown J, Medley N, Dalziel SR. Antenatal corticosteroids for accelerating fetal lung maturation for women at risk of preterm birth. Cochrane Database Syst Rev 2017;3:CD004454

9 American College of Obstetricians and Gynecologists. COVID-19 FAQs for obstetricians-gynecologists, obstetrics. Washington, DC: ACOG; 2020. Available at: https://www.acog.org/clinical-information/physician-faqs/covid-19-faqs-for-ob-gyns-obstetrics. American College of Obstetricians and Gynecologists: American Journal of Obstetrics and Gynecology. Accessed May 29, 2020

10 McIntosh JJ. Corticosteroid guidance for pregnancy during COVID19 pandemic. Am J Perinatol 2020;37(08):809-812

11 Chen H, Guo J, Wang C, et al. Clinical characteristics and intrauterine vertical transmission potential of COVID-19 infection in nine pregnant women: a retrospective review of medical records. Lancet 2020;395(10226):809-815

12 Coronado Munoz A, Nawaratne U, McMann D, Ellsworth M, Meliones J, Boukas K. Late-onset neonatal sepsis in a patient with covid-19. N Engl J Med 2020;382(19):e49

13 Zeng H, Xu C, Fan J, et al. Antibodies in infants born to mothers with COVID-19 pneumonia. JAMA 2020;323(18):1848-1849

14 Wang W, Xu Y, Gao R, et al. Detection of SARS-CoV-2 in different types of clinical specimens. JAMA 2020;323(18):1843-1844

15 Shah PS, Diambomba Y, Acharya G, Morris SK, Bitnun A. Classification system and case definition for SARS-CoV-2 infection in pregnant women, fetuses, and neonates. Acta Obstet Gynecol Scand 2020;99(05):565-568

16 Baud D, Greub G, Favre G, et al. Second-trimester miscarriage in a pregnant woman with SARS-CoV-2 infection. JAMA 2020;323 (21):2198-2200

17 Yang Z, Wang M, Zhu Z, Liu Y. Coronavirus disease 2019 (COVID19) and pregnancy: a systematic review. J Matern Fetal Neonatal Med 2020:1-4

18 Alzamora MC, Paredes T, Caceres D, Webb CM, Valdez LM, La Rosa M. Severe COVID-19 during pregnancy and possible vertical transmission. Am J Perinatol 2020;37(08):861-865

19 Zamaniyan $M$, et al. Preterm delivery in pregnant woman with critical COVID-19 pneumonia and vertical transmission. Prenat Diagn 2020

20 Mimouni F, Lakshminrusimha S, Pearlman SA, Raju T, Gallagher PG, Mendlovic J. Perinatal aspects on the covid-19 pandemic: a practical resource for perinatal-neonatal specialists. J Perinatol 2020;40(05):820-826

21 Sichitiu J, Fakhouri F, Desseauve D. Antenatal corticosteroid therapy and COVID-19: Pathophysiological considerations. Acta Obstet Gynecol Scand 2020;99(07):952 